\title{
Homemade-device-induced negative pressure promotes wound healing more efficiently than VSD-induced positive pressure by regulating inflammation, proliferation and remodeling
}

\author{
JINYAN LIU ${ }^{1}$, FENG HU ${ }^{2}$, JINTIAN TANG ${ }^{3}$, SHIJIE TANG ${ }^{4}$, KUN XIA ${ }^{5}$, SONG WU $^{6}$, CHAOQI YIN ${ }^{2}$, \\ SHAOHUA WANG ${ }^{2}$, QUANYONG HE ${ }^{2}$, HUIQING XIE ${ }^{7}$ and JIANDA ZHOU ${ }^{2}$
}

\begin{abstract}
${ }^{1}$ Department of Nuclear Medicine, The First Xiangya Hospital of Central South University, Changsha, Hunan 410008;
${ }^{2}$ Department of Plastic Surgery, The Third Xiangya Hospital of Central South University, Changsha, Hunan 410013;

${ }^{3}$ Institute of Medical Physics and Engineering, Tsinghua University, Beijing 100084; ${ }^{4} \mathrm{Cleft}$ Lip and Palate Treatment Center,

Second Affiliated Hospital, Shantou University Medical College, Shantou, Guangdong 515041; ${ }^{5}$ Key Laboratory of

Medical Information Research, Central South University, Changsha, Hunan; Departments of ${ }^{6}$ Orthopedics and

${ }^{7}$ Rehabilitation Medicine, The Third Xiangya Hospital of Central South University, Changsha, Hunan 410013, P.R. China
\end{abstract}

Received August 17, 2015; Accepted February 22, 2017

DOI: $10.3892 / \mathrm{ijmm} .2017 .2919$

\begin{abstract}
Vacuum sealing drainage (VSD) is an effective technique used to promote wound healing. However, recent studies have shown that it exerts positive pressure (PP) rather than negative pressure (NP) on skin. In this study, we created a homemade device that could maintain NP on the wound, and compared the therapeutic effects of VSD-induced PP to those of our homemade device which induced NP on wound healing. The NP induced by our device required less time for wound healing and decreased the wound area more efficiently than the PP induced by VSD. NP and PP both promoted the inflammatory response by upregulating neutrophil infiltration and interleukin (IL)- $1 \beta$ expression, and downregulating IL-10 expression. Higher levels of epidermal growth factor (EGF), transforming growth factor (TGF)- $\beta$ and platelet-derived growth factor (PDGF), and lower levels of basic fibroblast growth factor (bFGF) were observed in the wound tissue treated with NP compared to the wound tissue exposed to PP. Proliferation in the wound tissue exposed to NP on day 10 was significantly higher than that in wound tissue exposed to PP. NP generated more fibroblasts, keratinized stratified epithelium, and less epithelia with stemness than PP. The levels of ccollagen I and III were both decreased in both the NP and PP groups. NP induced a statistically significant increase in the expression of fibronectin $(\mathrm{FN})$ on days 3 and 10 compared to PP. Furthermore, the level of matrix metalloproteinase (MMP)-13 increased in the NP group, but
\end{abstract}

Correspondence to: Professor Jianda Zhou, Department of Plastic Surgery, The Third Xiangya Hospital of Central South University, 138 Tongzipo Road, Changsha, Hunan 410013, P.R. China E-mail: zhoujianda_csu@163.com

Key words: wound healing, negative pressure, cell infiltration, positive pressure, vacuum sealing drainage, growth factor, inflammation, extracellular matrix decreased in the PP group on day 3. NP also induced a decrease in the levels of tissue inhibitor of metalloproteinase (TIMP)-1 and TIMP-2 during the early stages of wound healing, which was significantly different from the increasing effect of PP on TIMP-1 and TIMP-2 levels at the corresponding time points. On the whole, our data indicate that our homemade device which induced NP, was more efficient than VSD-induced PP on wound healing by regulating inflammation, secretion, proliferation and the distribution of different cells in wound tissue.

\section{Introduction}

Wound healing is a dynamic and complex process through which the skin repairs itself following injury. It involves a series of coordinated phases, including hemostasis, inflammation, proliferation and remodeling (1). The inflammatory response is essential for removing the bacteria and cell debris from the wound. Additionally, different types of cells coordinate to influence wound repair and re-establish barrier function (2). Epithelial cells migrate across the surface of the wound bed and form a new epithelial layer (3). Mesenchymal stem cells (MSCs) play an important role in the inflammation, proliferation and remodeling phase of normal wound healing (4). In the proliferation phase, revascularization of the wound bed is a crucial stage of wound healing, which can reconstruct the circulation and nutrition supplement in the wound tissue. Therefore, endothelial cells are also needed to play an important role to break through the dermis of the wound and form tubes in the newly developing tissue $(5,6)$. In the final stage of wound healing, fibroblasts are attracted into the wound bed and begin to secrete collagenous extracellular matrix (ECM) that constitutes the newly formed granulation tissue, thereby providing structural integrity to the wound (5). Remodeled collagen promotes the maturation of the wound.

It is known that mechanical stress can influence wound healing by affecting the behavior of cells within the dermis (3). In wound treatment, vacuum sealing drainage (VSD), also known 
as negative pressure (NP) wound therapy, has been extensively researched (7) and has been proven to be markedly effective in wound healing $(8,9)$. VSD has been certified to decrease bacterial colony formation $(10,11)$, edema $(12,13)$, the permeability of vessels $(11,14,15)$, and to increase angiogenesis and blood flow to the wound margins $(12,13)$. It was originaly considered that VSD exerts NP on the wound. However, in recent years, some researches have demonstrated that VSD exerts positive pressure (PP) on the wound rather than NP (16). In the present study, we created a homemade device and found that it could maintain NP on the wound. We wished to determine whether VSD-induced PP or the NP induced by our homemade device was more efficient on wound healing.

In this study, we examined the effects and mechanisms of PP and NP using an animal wound healing model. The infiltration of inflammatory cells and growth factors were first analyzed. The distribution scale of different cell types in wound tissue following treatment with the pressure devices was then detected by histological analysis, including endothelial cells, MSCs, epithelial cells and fibroblasts. Furthermore, different types of collagen deposition were measured by western blot analysis. Our data demonstrate that the NP induced by our homemade device was more effective in promoting wound healing than the VSP-induced PP.

\section{Materials and methods}

Ethics statement. All methods used in this study were carried out in accordance with the approved ethical guidelines of Central South University. The study protocol was approved by the Central South University Institutional Review Board.

Homemade NP device. The NP device with a cavity was made with medical silica gel by our laboratory. Briefly, we firstly used paraffin to carve a model with a square-hat-like structure. The model was then placed in a glass container, and surrounded by dental gypsum suspension (special plaster used in the Department of Stomatology). After the plaster water had evaporated, the paraffin model was dissolved and removed in hot water to obtain the plaster mold (Fig. 1A). Subsequently, the medical silica gel A and B components were mixed at the proportion of 1:1, and injected into the plaster mold. After being shaped at room temperature, the plaster mold was removed, and the silicone NP device (Fig. 1B; length, $4.5 \mathrm{~cm}$; width, $3.5 \mathrm{~cm}$; height, $2 \mathrm{~cm}$ ) was ready for use in the following experiments.

The profile of the homemade silicone NP device could be punctured by an infusion needle which was connected to a portable suction pump with an accurate pressure gauge (AZ 8205; AZ Instrument Corp., Taichung, Taiwan) with a tube, and can aspirate a pressure of $-37.5 \mathrm{mmHg}$ (also known as $-5 \mathrm{kPa}$ ). The pressure beneath the homemade device was measured by an intracranial tissue pressure microsensor (Codman; Johnson and Johnson Professional, Inc., Raynham, MA, USA) (16), which is pasted on the skin surface and is small enough to be covered by the homemade device. It measures both PP and NP in gas and liquids or any compliant substances, such as soft tissue. The cavity was sealed to maintain the corresponding pressure.

The VSD device was used as a control device in this study (Fig. 1C) (RENASYS; Smith \& Nephew, Inc., Andover,
MA, USA). VSD is a type of device that has been widely used in the treatment of trauma wounds. The device is comprised of a transparent film with the permeability of biological seal, a drainage tube and drainage device.

The main difference between the VSD and our homemade device was that the material used was different, and that there was no cavity between the VSD and the skin, as opposed to the cavity with our homemade device. In other words, the VSD is placed close onto the wounded skin and has a vacuum suction effect, while the homemade device is not placed so close to the skin due to the existence of a cavity beneath the device.

Animal care and surgical procedure. The skin trauma model was established using 12 female New Zealand rabbits (purchased from SLACCAS, Shanghai, China) each weighing 2.5-3.0 kg. The dorsum of the animal was cleaned, shaved and treated with Veet (Reckitt Benckiser, Parsippany, NJ, USA) for hair removal. After weighing the animals, $1 \mathrm{ml} / \mathrm{kg} 3 \%$ pentobarbital (P3761; Sigma, St. Louis, MO, USA) sodium was injected intravenously for anesthetization. A circular area of $1 \mathrm{~cm}$ in diameter was marked using a pen at both sides of the back of the rabbits. After disinfecting with chlorhexadine and alcohol 3 times, lidocaine hydrochloride (H37022147; Shandong Hualu Pharmaceutical Co., Ltd., Liaocheng, China) was injected subcutaneously in the circular area mentioned above. Subsequently, a sterile surgical blade was used to make a surgical incision on the skin along the marked line, followed by blunt separation of the subcutaneous superficial layer of the fascia using sterile scissors. Finally, the $1 \mathrm{~cm}$-diameter-circular skin was fully cut off to construct the wound model of full-thickness skin defect.

Corresponding therapies were randomized on the left or right side of the animal wound (12 for the control group and 12 for the experimental group). For the control group, each site was disinfected with iodine and alcohol complex 3 times each day. For the experimental group, as shown in Fig. 1D, each site was covered by the homemade NP device mentioned above or the VSD device, and pumped using a vacuum device to maintain a pressure of $-37.5 \mathrm{mmHg}$. The pressure that the skin received was detected using the intracranial tissue pressure microsensor and monitored using an ICP express monitoring system (Codman; Johnson and Johnson Professional, Inc.). The animals of experimental groups receive a 2 -h treatment each day for 7 days. The rabbits were euthanized at tbhe indicated time points (days 3, 7, 10 and 14), and the complete area of the wounds was harvested and fixed in $4 \%$ paraformaldehyde for use in histological analysis.

Immunohistochemical analysis. Ki67 was used to determine cell proliferation; CD31 for endothelial cells; CK10 for epithelial subset; and Masson's staining for collagen fibers. The rabbits were euthanized at the indicated time points. The wounds were excised and fixed in $4 \%$ paraformaldehyde overnight at $4^{\circ} \mathrm{C}$, embedded in paraffin, divided into sections and stained with antibodies to CK10 (bs-11186R; Bioss, China), CD31 (ab199012; Abcam, Cambridge, USA) and Ki67 (ab15580; Abcam), or Masson's trichrome stain (ab150686; Abcam), and imaged using an Olympus BX50 microscope with a DP71 camera and Software cellSens Standard 1.6 (Olympus Corp., Center Valley, PA, USA). The integrated option density (IOD) of the digital images was evaluated using Image-Pro Plus software 5.1 (Media Cybernetics, Inc, Rockville, MD, USA). 

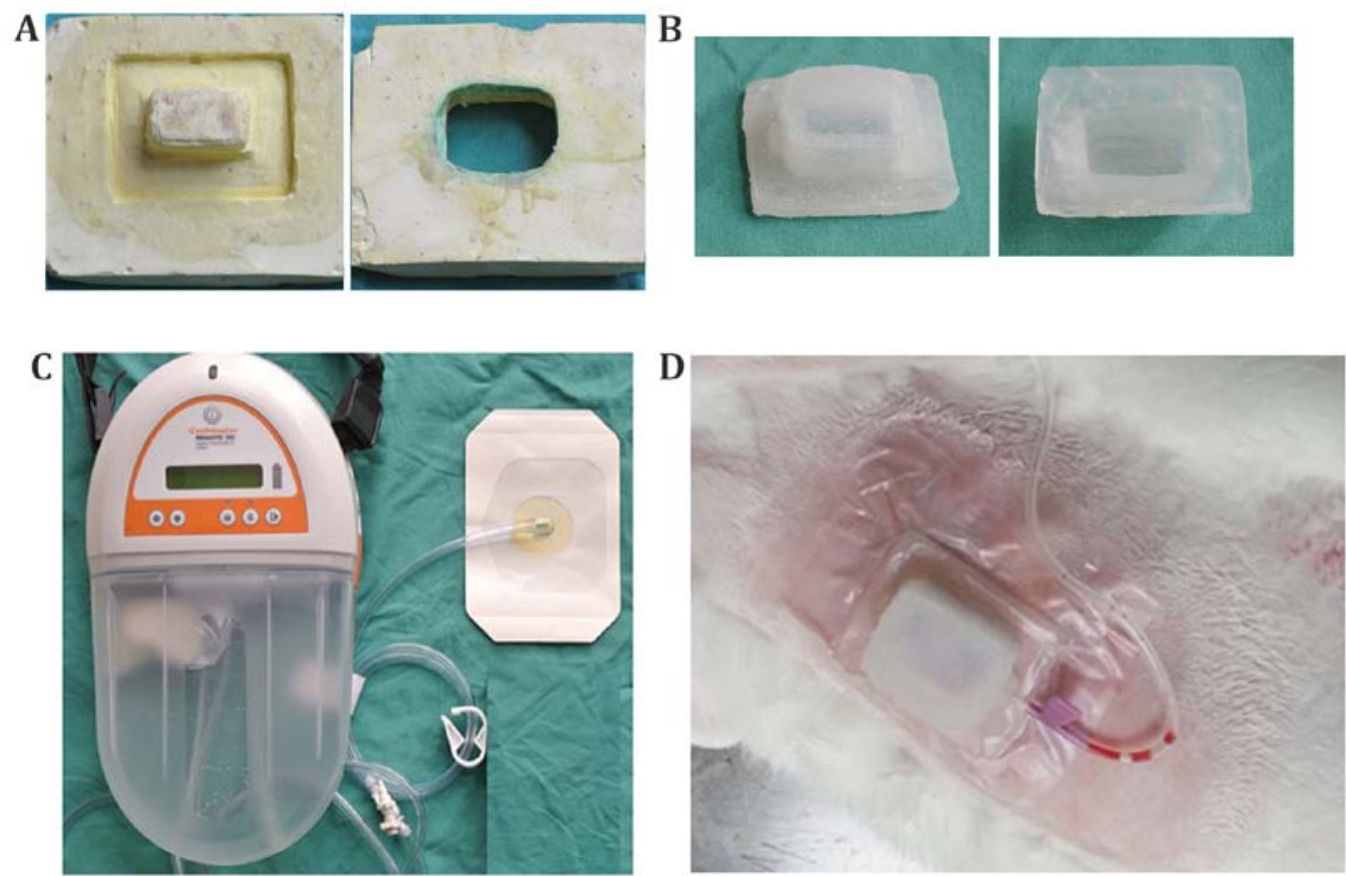

Figure 1. Construction of homemade NP device. (A) Representative images of plaster-made mold of homemade NP device, including the bottom and upper part (B) Representative images of medical silicon homemade device. (C) Representative images of the VSD device. (D) Treatment of animals with wounds with our homemade device. The wound was covered with our homemade device, and an infusion needle was punctured into the device cavity and connected to a portable suction pump. NP, negative pressure; VSD, vacuum sealing drainage.

Immunofluorescence staining. CD29 and CD90 were used to label MSCs; CK14 and CK19 for different epithelial subsets; and S-100A4 for fibroblasts. The rabbits were euthanized at the indicated time points. The wounds were soaked in $30 \%$ sucrose in PBS overnight at $4^{\circ} \mathrm{C}$ and then embedded in Tissue-Tek O.C.T (Sakura Finetek USA, Inc., Torrance, CA, USA). Serial $8 \mathrm{~mm}$ thick sections were cut at $-20^{\circ} \mathrm{C}$ and placed on poly-L-lysine-coated microscopic slide. Non-specific labeling was blocked with $10 \%$ normal goat serum for $30 \mathrm{~min}$ at room temperature. The sections were incubated with primary antibodies against CD29 (bs-3973R; Bioss), CD90 (ab225; Abcam), CK14 (ab77684; Abcam), CK19 (ab84632; Abcam), and S-100A4 (bs-3759R; Bioss) at $4^{\circ} \mathrm{C}$ overnight. Omitting primary antibodies was performed as control staining. After several washes, the secondary antibodies (ab150077; Abcam) were applied followed by incubation for $60 \mathrm{~min}$ at room temperature. An Olympus BX51 microscope equipped with fluorescence and a CCD camera was used to capture the fluorescence images. The IOD was evaluated using Image-Pro Plus 5.1 software (Media Cybernetics, Inc.).

Quantitative polymerase chain reaction ( $q P C R$ ) for the analysis of $m R N A$ expression. Total RNA was extracted from the wound tissues at different time points with the use of TRIzol reagent (Life Technologies, Shanghai, China) according to the manufacturer's instructions. cDNA was synthesized using the TaqMan MicroRNA Reverse Transcription kit (Applied Biosystems Life Technologies, Foster City, CA, USA). qPCR was performed with the use of a fluorescence quantitative PCR instrument (ABI 7500 thermocycler; Life Technologies) and SYBR-Green Universal PCR Master Mix (Bio-Rad, Hercules, CA, USA). The oligonucleotide sequences of the primer sets used were as follows: interleukin (IL)-1 $\beta$ (sense, ACC AAC
AAG TGG TGT TCT CC; antisense, TCT TTG GGT AAC GGT TGG GG), IL-10 (sense, TCA CCG ATT TCT CCC CTG TG; antisense, GAA GAT GTC AAA CTC ACT CAT GC), basic fibroblast growth factor (bFGF) (sense, AGC GGC TGT ACT GCA AAA AC; antisense, AAC GGT TTG CAC ACA CAC CT), epidermal growth factor (EGF) (sense, AGT GCT CGT ATG TGC TCT TGT G; antisense, ATT CTA ACC ATT TCC TTC CCA GT), vascular endothelial growth factor (VEGF) (sense, GCC AGC ACA TAG GGG AGA TG; antisense, GCT TTC GTT TTT GCC CCT T), insulin-like growth factor-1 (IGF-1) (sense, CAT GCC CAA GAC TCA GAA GT; antisense, CAA ATG TAC TTC CTT TCC TTC TC), platelet-derived growth factor (PDGF) (sense, AAG TGT GAG ACG GTG GCA G; antisense, TGT GCT TGA ACT TGT GGT GC), transforming growth factor (TGF)- $\beta$ (sense, ACA GCA TGA ACC GAC CCT TC; antisense, GGT CCT TGC GGA AGT CAA TG), GAPDH (sense, TTT GTG ATG GGC GTG AAC C; antisense, CCC TCC ACA ATG CCG AAG T). PCR was performed in a total volume of $20 \mu \mathrm{l}$, including $10 \mu \mathrm{l}$ of 2X SYBR-Green qPCR Mix, $1 \mu \mathrm{l}$ of each forward and reverse primer $(10 \mu \mathrm{mol} / \mathrm{l}), 1 \mu \mathrm{l}$ each cDNA sample, and $7 \mu \mathrm{l}_{2} \mathrm{O}$. Amplifications were carried out in triplicate in 96-well microtiter plates. The thermal cycling conditions were as follows: $95^{\circ} \mathrm{C}$ for $5 \mathrm{~min}$, followed by 65 cycles of $95^{\circ} \mathrm{C}$ for $10 \mathrm{sec}$, and $60^{\circ} \mathrm{C}$ for $10 \mathrm{sec}$, and finally followed by $72^{\circ} \mathrm{C}$ for $10 \mathrm{sec}$.

Western blot analysis. Western blot analysis was performed to detect the protein expression levels. In brief, tissues and cells were solubilized in cold RIPA lysis buffer. Proteins were separated by $10 \%$ SDS-PAGE, and transferred onto PVDF membranes, which were then incubated with PBS containing 5\% milk overnight at $4^{\circ} \mathrm{C}$. The PVDF membranes were then incubated with primary antibodies [collagen I, 

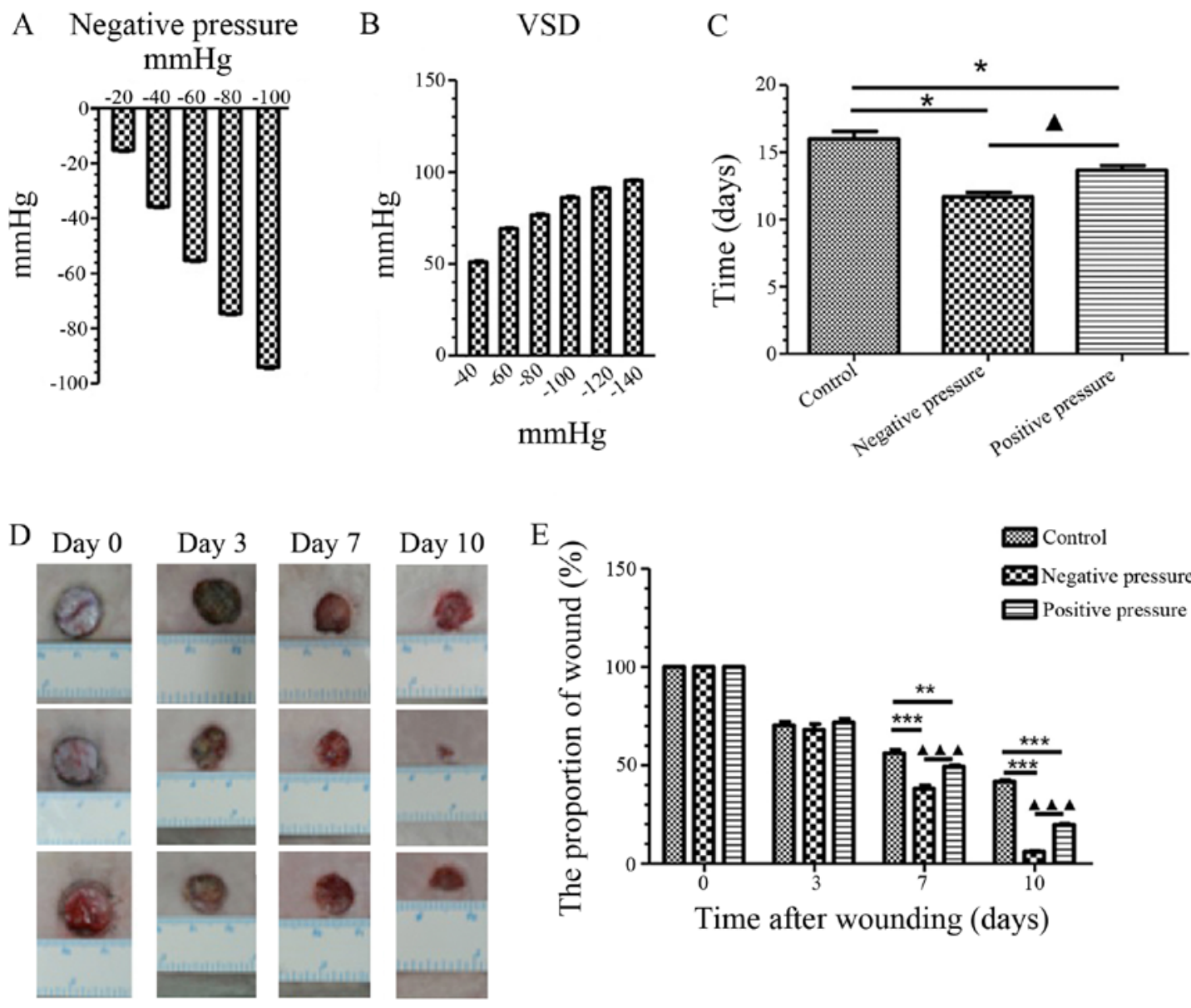

Figure 2. NP promotes wound healing and reduces the wound proportion area more efficiently than PP. (A) Detection of pressure on skin that was induced by the homemade device on wound healing. (B) Detection of pressure on skin that was induced by the VSD. (C) Statistical analysis of the required time for wound healing in the different treatment groups. Data are presented as the means $\pm \mathrm{SEM}, \mathrm{n}=3$. ${ }^{*} \mathrm{P}<0.05$, compared with the control; ${ }^{\wedge} \mathrm{P}<0.05$, compared with $\mathrm{NP}$. (D) Representative images of wound healing on days $0,3,7$, and 10 in the different treatment groups. (E) Statistical analysis of the wound proportion area in the different treatment groups. Data are presented as the means $\pm \mathrm{SEM}, \mathrm{n}=3 .{ }^{* * *} \mathrm{P}<0.01,{ }^{* * *} \mathrm{P}<0.001$, compared with the control; ${ }^{\wedge}{ }^{\wedge} \mathrm{P}<0.001$, compared with NP. NP, negative pressure; PP, positive pressure; VSD, vacuum sealing drainage.

(2 $\mu \mathrm{g} / \mathrm{ml}$, ab6308); collagen III (1:8,000, ab7778); FN, (3 mg/ ml, ab6328); MMP13 (1 $\mu \mathrm{g} / \mathrm{ml}$, ab84594); TIMP-1 (0.2 $\mu \mathrm{g} /$ ml, ab126847); TIMP-2 (1:1,000, ab1828); all from Abcam] at room temperature for $3 \mathrm{~h}$, respectively, and then secondary antibodies [goat anti-mouse (ab6785, 1:10,000); goat anti-rabbit (ab6721, 1:20,000); donkey anti-goat (ab6881, 1:10,000); all from Abcam] at room temperature for $1 \mathrm{~h}$. An ECL kit was then used to perform chemiluminent detection. Relative protein expression was analyzed using Image-Pro Plus software 6.0, represented as the density ratio vs. GAPDH.

Statistical analysis. All experiments were repeated 3 times. All data are presented as the mean values \pm SEM. Two-way ANOVA was used to compare the differences between groups. A value of $\mathrm{P}<0.05$ was considered to indicate a statistically significant difference.

\section{Results}

Homemade device-induced NP promotes wound healing and reduces the wound proportion area more efficiently than $V S D$-induced PP. We first measured the pressure generated by the homemade NP device and VSD, and found that the home- made NP device generated NP on the skin (Fig. 2A), whereas the VSD device exerted PP on the skin (Fig. 2B). Additionally, we compared the effects of NP and PP on wound healing. As shown in Fig. 2C, both types of pressure efficiently promoted wound healing compared with the controls (NP, $\mathrm{P}<0.05$; $\mathrm{PP}, \mathrm{P}<0.05)$. Of note, NP was shown to promote wound healing within a shorter period of time compared to PP $(\mathrm{P}<0.05)$. Additionally, the wound healing rate was measured at different time points during wound healing (Fig. 2D). The proportion of wound area was significantly reduced in both pressure groups compared to the control group on day 7 (NP, $\mathrm{P}<0.001$; $\mathrm{PP}, \mathrm{P}<0.01)$ and day 10 (NP, $\mathrm{P}<0.001$; $\mathrm{PP}, \mathrm{P}<0.001)$; however, exposure to NP led to a more significant reduction in the wound proportion area than $\mathrm{PP}(\mathrm{P}<0.001)$ (Fig. $2 \mathrm{E})$. On the whole, NP more efficiently promoted wound healing than PP.

$N P$ and PP promote the inflammatory response and the expression of growth factors in wound healing. The infiltration of macrophages and neutrophils was examined by tissue analysis following exposure to NP or PP (Fig. 3A). We found that the infiltration of macrophages was significantly reduced from days 3 to 10 in both the NP and PP treatment goups compared to the controls (Fig. 3B). The number of neutrophils 

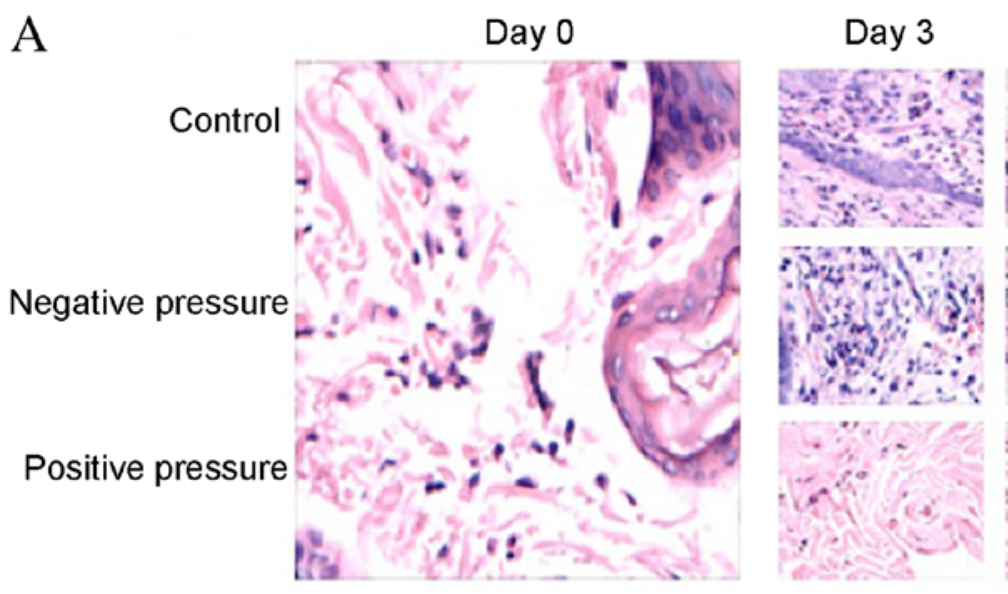

Day 7

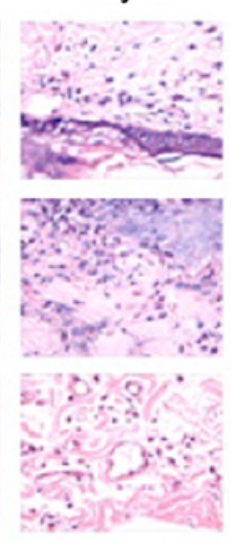

Day 10

Day 14

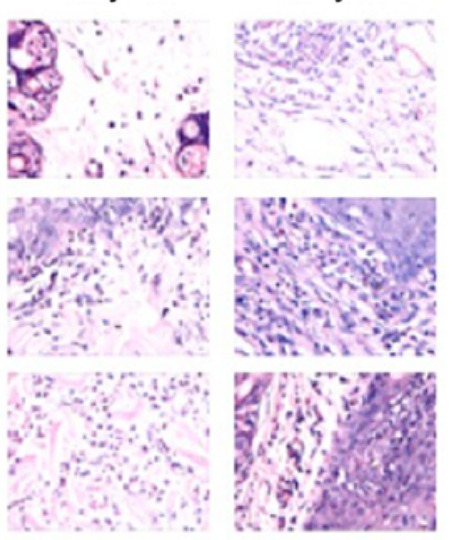

B

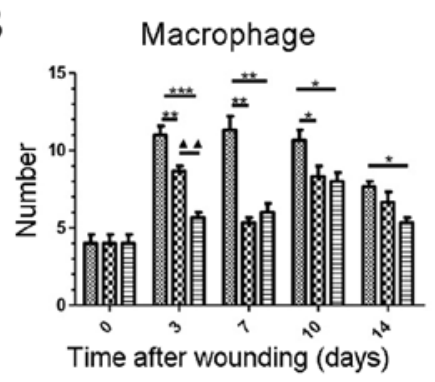

E

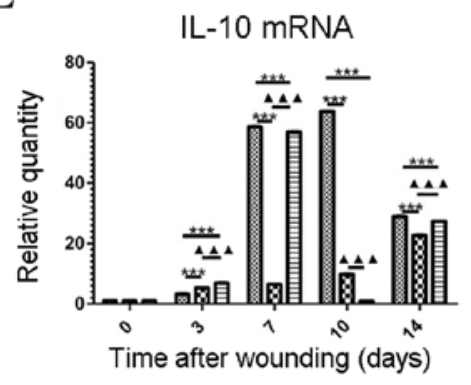

$\mathrm{H}$
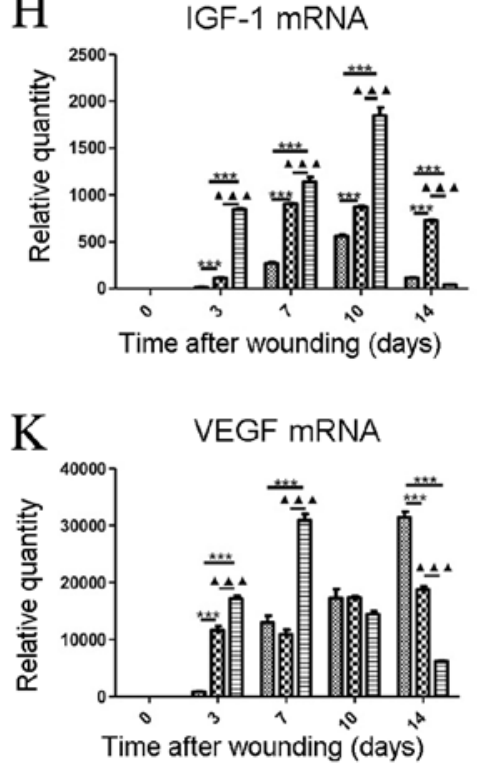

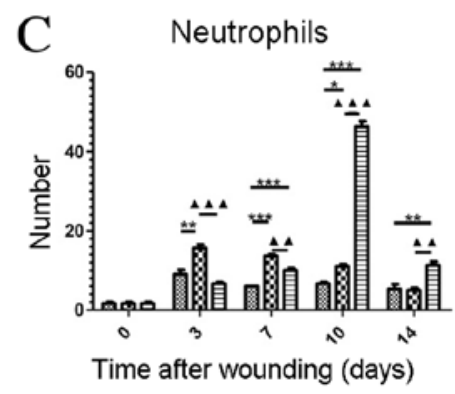

F
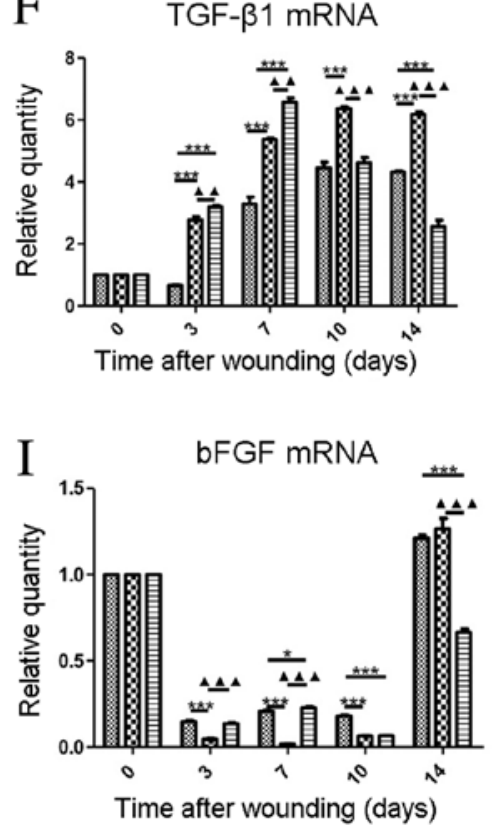

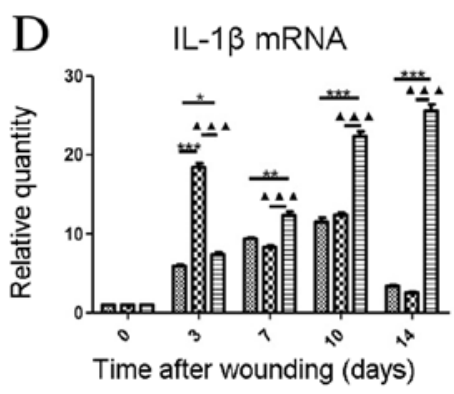

G
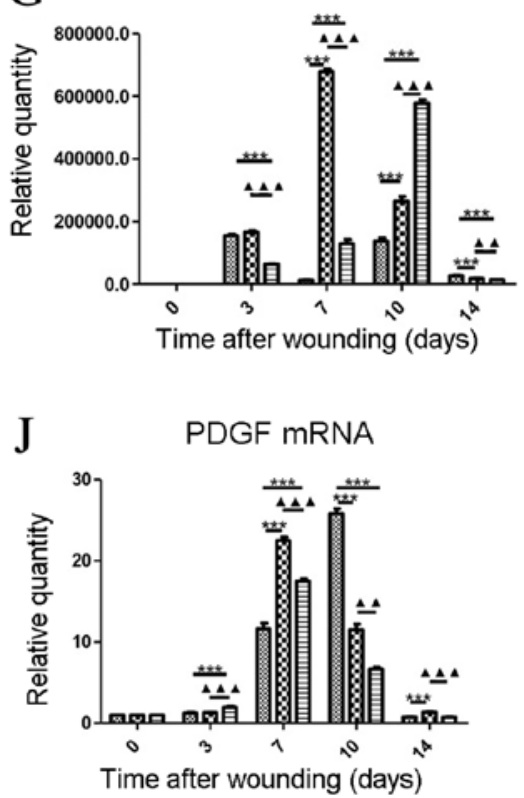

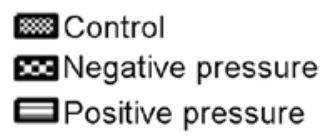

Figure 3. Analysis of inflammatory and growth factor secretion in wound tissue in the NP and PP groups. (A) Representative images of H\&E staining of inflammatory cells in wound tissues following pressure treatment on days 3, 7, 10, and 14. The upper lane indicates the control group, the middle lane indicates the NP group, and the lower lane indicates the PP group. (B) Statistical quantification of macrophage infiltration in wound tissue at different time points. (C) Statistical quantification of neutrophil infiltration in wound tissue at different time points. Statistical analysis of the mRNA expression of (D) IL-1 $\beta$, (E) IL-10, (F) EGF, (G) TGF- $\beta 1$, (H) IGF-1, (I) bFGF, (J) PDGF, (K) VEGF in wound tissue examined by qPCR. Data are presented as the means \pm SEM, $=3$ experiments. ${ }^{* * * *} \mathrm{P}<0.001,{ }^{* *} \mathrm{P}<0.01,{ }^{*} \mathrm{P}<0.05$, compared with the control. ${ }^{\wedge \wedge} \mathrm{P}<0.001,{ }^{\wedge} \mathrm{P}<0.01$, compared with NP. NP, negative pressure; $\mathrm{PP}$, positive pressure. 

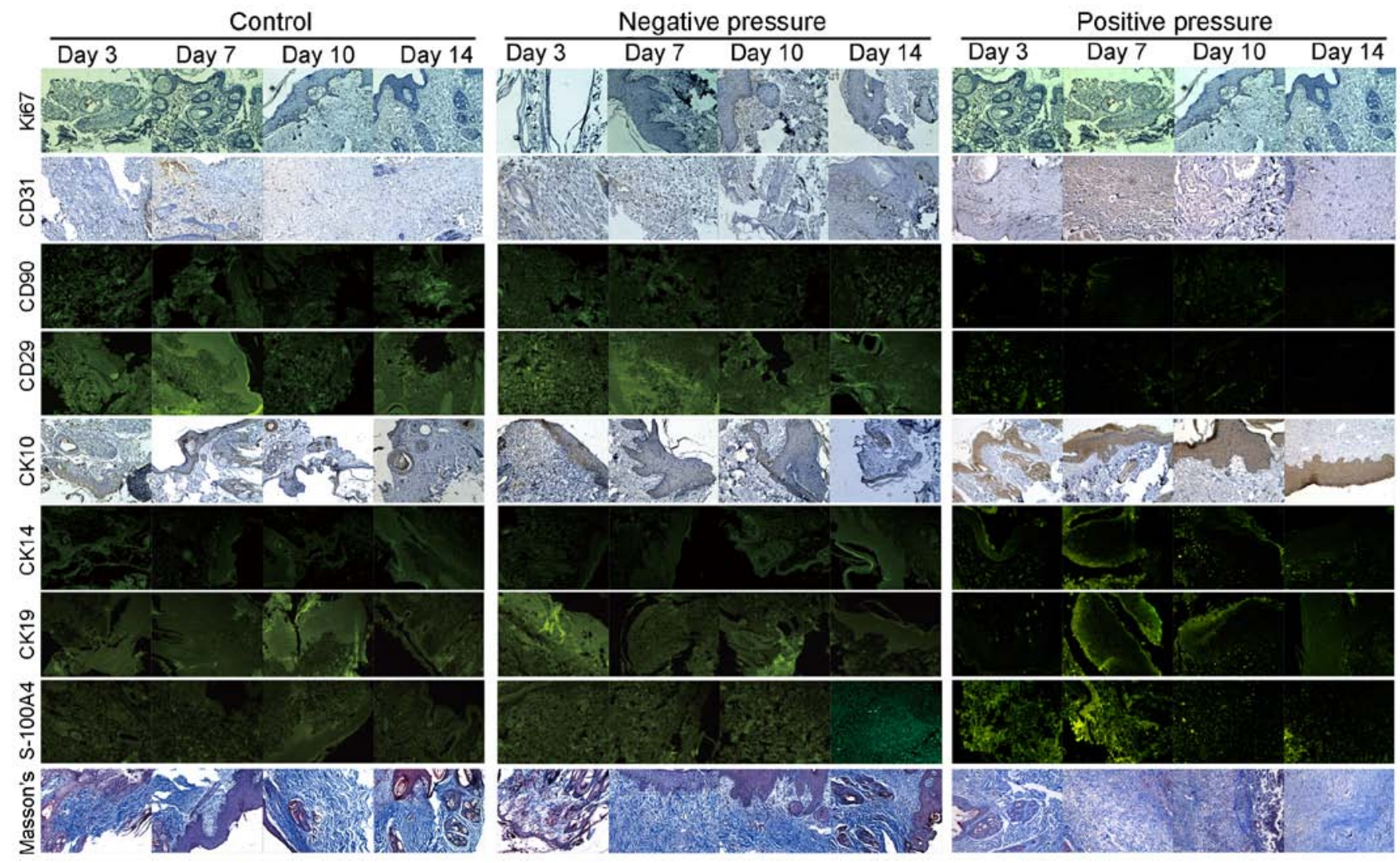

Figure 4. Histological analysis of different cell markers in wound tissue in the different pressure treatment groups. Representative images of H\&E staining and immunofluorescence staining in wound tissues following pressure treatment on days 3, 7, 10, and 14. Ki67, CD31, CD90, CD29, CK10, CK14, CK19, S-100A4, and Masson's staining were all used for histological analysis.

was significantly increased by NP from day 3 compared with the controls (day $3, \mathrm{P}<0.01$; day $7, \mathrm{P}<0.001$; day $10, \mathrm{P}<0.05$ ); however, in the PP group, the number of neutrophils was increased from day 7 compared to the controls (Fig. 3C). Subsequently, inflammation-related factors were analyzed in the wound tissue exposed to NP and PP. During the early stages of wound healing (from day 0 to day 10), NP treatment promoted the expression of pro-inflammatory IL-1 $\beta$ on day 3 , which was earlier than PP (Fig. 3D). The expression of antiinflammatory factor IL-10 was only increased on day 3 in both the NP and PP groups (Fig. 3E). TGF- $\beta$ was significantly upregulated in the NP and PP groups, although the effect of NP was more long lasting (Fig. 3F). As regards growth factors in the promotion of wound healing, the expression levels of EGF and IGF-1 were both upregulated in the NP and PP groups on days 7 and 10; however, the changes in bFGF expression were more complex (Fig. 3G-I). NP and PP both led to an earlier expression peak in PDGF and VEGF, although in the later stages of wound healing (day 14) a lower expression of VEGF was observed in the NP and PP groups than in the control (Fig. 3J and K). On the whole, NP and PP promoted the inflammatory response and the secretion of growth factors in the earlier stages of wound healing.

Changes in the proliferation and distribution of different cell types in wound tissue exposed to NP and PP. Tissue staining was carried out to examine the effects of NP and PP on the proliferation and distribution of different cell types in the wound tissue. As shown in Fig. 4, Ki67 was used to detect cell proliferation, endothelial cells were labeled with CD31, while MSCs were labeled with CD90 and CD29; CK10, CK14 and CK19 were used to stain the different epithelial cell subsets; S-100A4 and Masson's trichrome staining were used to mark the fibroblasts and collagen fibers. The quantified results of the histological analysis in Fig. 4 are all shown in Fig. 5. The expression of Ki67 was used for the detection of proliferation. As shown in Fig. 5A, NP and PP both significantly increased cell proliferation in the wound tissue on day 10 compared to the controls (NP, $\mathrm{P}<0.001 ; \mathrm{PP}, \mathrm{P}<0.01$ ); however, the effect of NP on proliferation was more potent than that of $\mathrm{PP}(\mathrm{P}<0.001)$. There was no significance among the groups at the other time points examined. These data indicated that the wound tissue was in a proliferative state, and that exposure to NP led to significantly greater proliferation on day 10 , as evidenced by the increase in Ki67 expression.

We then measured the number of endothelial cells in wound tissue by CD31-positive staining. As shown in Fig. 5B, the IOD value of CD31 was significantly increased in the NP and PP groups compared with the controls from day $3(\mathrm{P}<0.001)$; however, PP increased the number of CD31-positive cells more significantly than NP in the wound tissue from days 3 to $10(\mathrm{P}<0.001)$.

The number of MSCs in the wound tissue was then detected by CD29- and CD90-double-positive staining. Our data indicated that the CD29- and CD90-positive area in the NP group was significantly reduced at the later stage of healing, while 


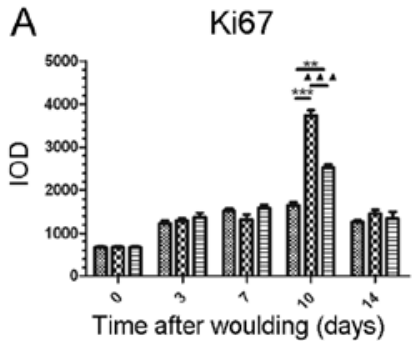

$\mathrm{E}$
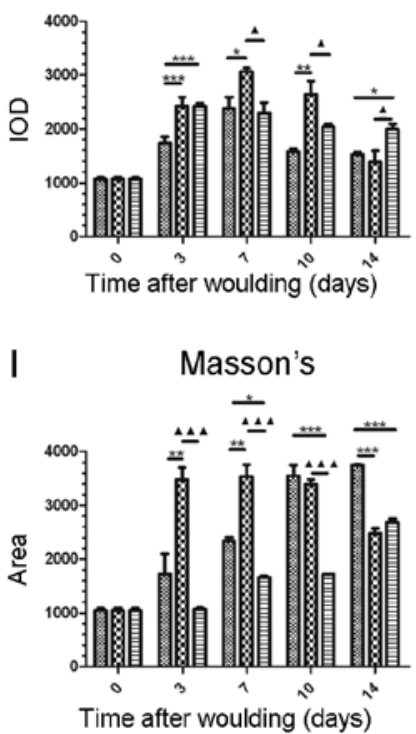

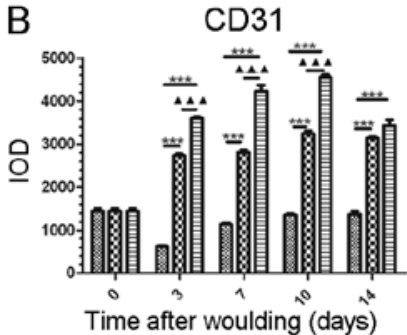

C
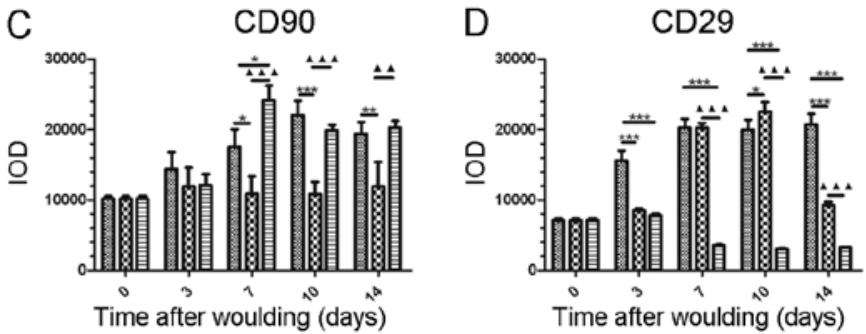

$\mathrm{F}$

CK14

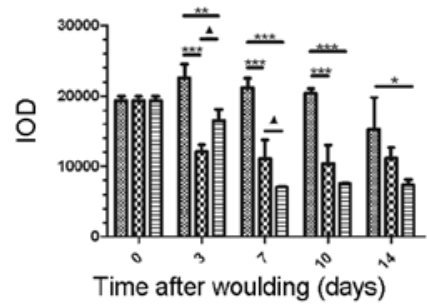

G

CK19
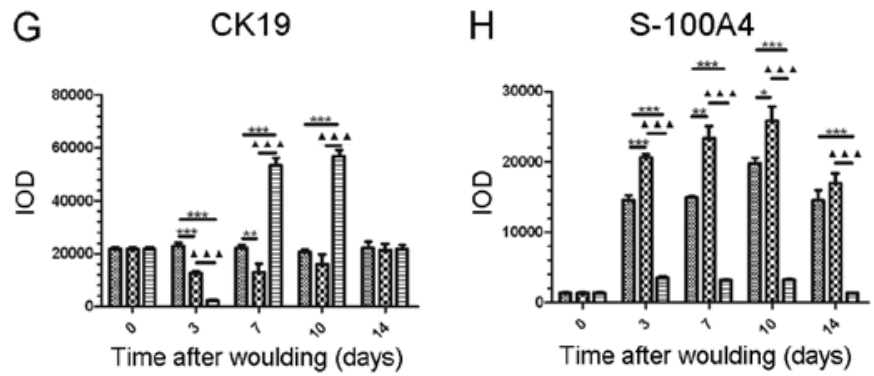

Figure 5. Statistical analysis of the histological results of different cell markers in wound tissue in the different pressure treatment groups. Statistical quantification of the IOD value of (A) Ki67, (B) CD31, (C) CD90, (D) CD29, (E) CK10, (F) CK14, (G) CK19, (H) S-100A4, (I) Masson's staining. Data are presented as the means \pm SEM, $n=3$ experiments. ${ }^{* * * *} \mathrm{P}<0.001,{ }^{* * *} \mathrm{P}<0.01,{ }^{*} \mathrm{P}<0.05$, compared with the control. ${ }^{\wedge \wedge}{ }^{\wedge} \mathrm{P}<0.001,{ }^{\wedge} \mathrm{P}<0.01$, compared with NP. IOD, integrated option density; NP, negative pressure.

the CD90-positive area in the PP group showed no reduction when compared with the control group from days 0 and 14 , although the CD29-positive area in the PP group was reduced from days 3 to day 14 (Fig. 5C and D). In general, a lower amount of MSCs was detected following exposure to NP in wound healing.

In order to examine the distribution of different epithelial cell subsets in wound healing, CK10, CK14 and CK19 were used to stain the slides. CK10 was mainly secreted by the keratinized stratified epithelium, and CK14 and CK19 were used to identify the basal layer epithelia and the epithelia with stemness, respectively. Our data demonstrated that NP induced a more significant increase in the number of CK10-positive cells in wound tissue from days 3 to 10; PP increased the number of CK10-positive cells more significantly on day 14 (Fig. 5E). Additionally, cells positive for CK14 were significantly decreased following exposure to NP and PP (Fig. 5F). PP induced a significant increase in the number of CK19-positive cells on days 7 and 10; however, NP induced a decreased in the number of CK19-positive cells on day 3 and 7 (Fig. 5G). This indicated that NP treatment generated more keratinized stratified epithelium, and less basal layer epithelia and epithelia with stemness in the early stages of wound healing, al no difference was observed on day 14. PP treatment led to an increase of epithelia with stemness in the early stages of wound healing, which differed from the effects of NP.

For the measurement of fibroblasts and collagen fibers in the wound tissue, S-100A4 and Masson's trichrome were used to stain the tissues, respectively. We found that NP significantly increased the proportion of fibroblasts $(\mathrm{P}<0.001)$ and collagen fibers $(\mathrm{P}<0.01)$ in wound tissue, while $\mathrm{PP}$ induced lowr amounts of these markers compared to NP $(\mathrm{P}<0.001)$ (Fig. 5H and I).

NP and PP modulate the generation of ECM components. In order to examine the structural recovery effect of NP and PP on wound healing, we analyzed the dermal papilla structure. As shown in Fig. 6A, the dermal papilla structure was flat and the arrangement of collagen fibers was disrupted in the 3 groups. Additionally, wound healing-related protein expression levels were measured following exposure to NP and PP (Fig. 6B). As shown in Fig. 6B-D, the levels of collagen I and III were significantly decreased in the NP and PP groups compared with the controls $(\mathrm{P}<0.001$ and $\mathrm{P}<0.001)$. NP induced a more significant increase in the expression of $\mathrm{FN}$ on day $3(\mathrm{P}<0.001)$ and day $10(\mathrm{P}<0.001)$ compared to $\mathrm{PP}$ (Fig. $6 \mathrm{~B}$ and $\mathrm{E})$. In addition, MMP-13 expression increased in the NP group $(\mathrm{P}<0.001)$, but decreased in the PP group $(\mathrm{P}<0.001)$ on day 3 (Fig. 6B and F). NP also induced a decrease in TIMP-1 $(\mathrm{P}<0.001)$ and 
A
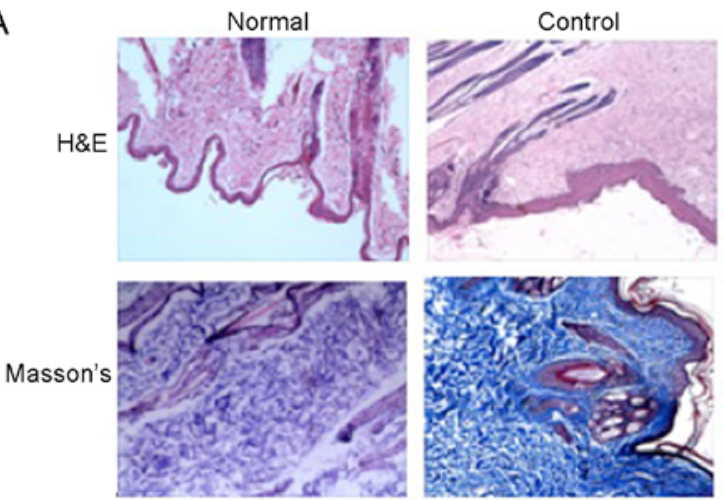

B

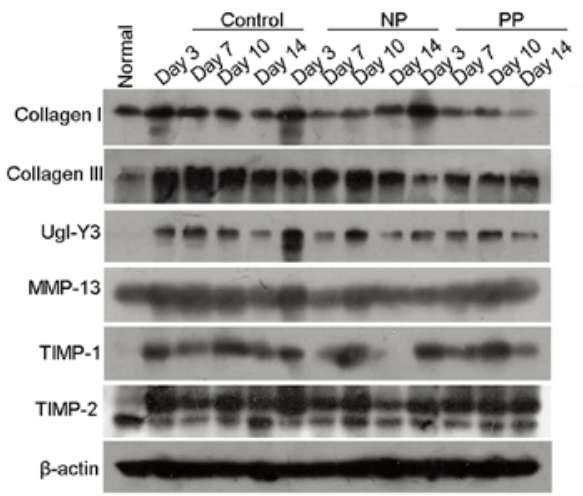

Negative pressure
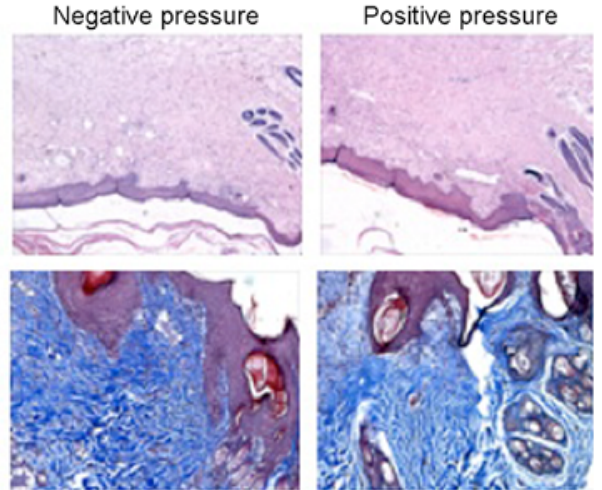
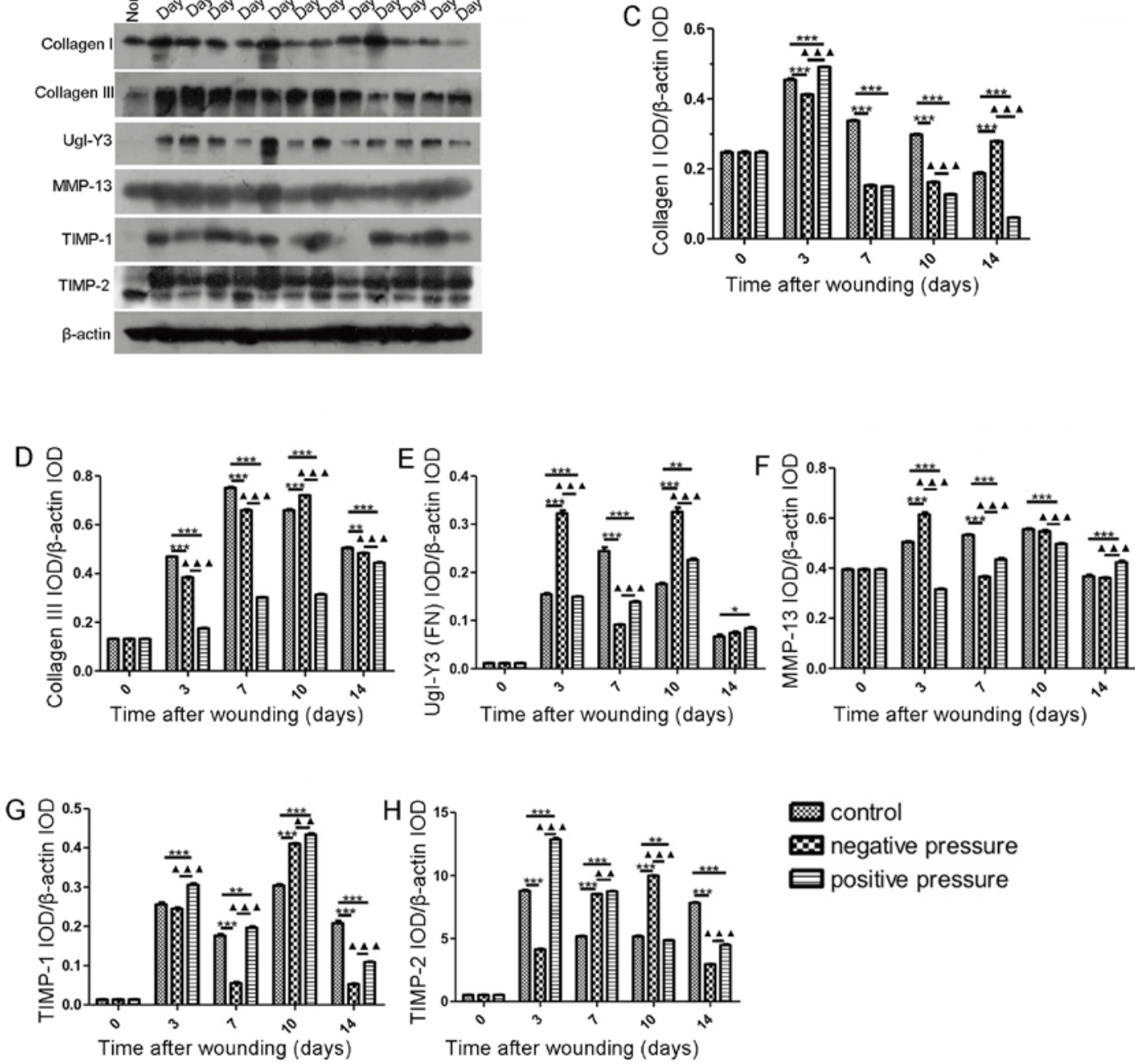

Figure 6. NP and PP affect the generation of extracellular matrix components. (A) Representative images of dermal papilla structure in the different pressure treatment groups. (B) Protein expression of different matrix components in wound tissues treated with different pressure types. Different time points (day 3, 7 , 10 and 14) were recorded. Statistical quantification of IOD value of (C) collagen I, (D) collagen III, (E) UgI-Y3 (FN), (F) MMP-13, (G) TIMP-1, (H) TIMP-2. Data are presented as the means $\pm \mathrm{SEM}, \mathrm{n}=3$ experiments. ${ }^{* * *} \mathrm{P}<0.001,{ }^{* *} \mathrm{P}<0.01,{ }^{*} \mathrm{P}<0.05$, compared with the control. ${ }^{\wedge \wedge} \mathrm{P}<0.001,{ }^{\wedge} \mathrm{P}<0.01$, compared with $\mathrm{NP}$. $\mathrm{NP}$, negative pressure; $\mathrm{PP}$, positive pressure; IOD, integrated option density.

TIMP-2 ( $\mathrm{P}<0.001)$ expression on days 7 and 3, respectively; this effect was significantly different from the upregulatory effect of PP on TIMP-1 ( $<<0.001)$ and TIMP-2 $(\mathrm{P}<0.001)$ at the corresponding time points (Fig. 6B, G and H). Collectively, NP and PP did not alter the dermal papilla structure and the arrangement of collagen fibers. NP induced a greater increase in MMP-13 expression and decreased TIMP-1/2 expression during the early stages of wound healing.

\section{Discussion}

The VSD technique is a therapeutic strategy which is widely used to promote rapid wound healing in traumatic soft wounds and chronic infections. In this study, we compared the VSD to a homemade device we created. The material used to create our homemade device differed from that used for the VSD. In addition, the main difference between the VSD and our homemade 
device in this study, was that in the VSD, there was no cavity between the device and the skin. As a result, the VSD is placed very close to the skin wound for vacuum suction, and the pressure on the skin is thus not negative, but positive (16). In other words, the pressure is perpendicular to the skin and is directed downwards. Additionally, as shown in our study, the existence of the cavity between the homemade device and the skin meant that NP was exerted on the skin after vacuum suction, the pressure being perpendicular to the skin and directed upwards. Furthermore, we examined the effects and differences between PP and NP on wound healing. Both types of pressure promoted the wound healing process and reduced the proportion of the wound. However, NP exerted more prominent wound healing effects. NP induced greater neutrophil infiltration and more pro-inflammatory factor secretion during the early stages of healing. Both NP and PP upregulated the expression of growth factors, such as EGF, VEGF, PDGF and TGF- $\beta 1$. Furthermore, we found that cell proliferation in the wound tissue was significantly increased by NP on day 10 . The distribution scale of different cell types and the generation of collagen in wound tissue were also altered following pressure treatment.

Inflammation is an essential stage of wound healing. Within hours of wounding, neutrophils are recruited to the wound site, and phagocytose debris and kill bacteria $(17,18)$. In this study, we detected the infiltration of neutrophils in wound tissues, and found that NP increased neutrophil infiltration to a greater extent than PP on day 3, which may enhance clearance at the early stages of wound healing and may be helpful in accelerating the healing process. In the NP group, the level of neutrophils from day 10 was similar to that of the controls, suggesting that this may help diminish the continuous or extended inflammation that often impedes the following stage of healing; this effect was confirmed by the results of the expression of IL- $1 \beta$ on day 10 and 14, which was similar between the control and NP group on these days. This was one of the key differences between NP and PP.

During the wound healing process, endothelial cells, fibroblasts and collagen significantly infiltrated the wound tissue following pressure treatment, while MSCs in the wound tissue in the NP group were lower in number compared to the control. In addition, NP promoted more keratinized stratified epithelium, and less basal layer of stratified epithelia and epithelia with stemness. These data are in accordance with those of other studies, that have proven that wound tissue treated with negative pressure wound therapy (NPWT) increases epithelial cell migration (19), endothelial migration $(20,21)$ and dermal fibroblast migration (22). Resident MSCs and circulating progenitor cells have also been observed to migrate into granulation tissue following the application of NPWT (23). Of note, the differentiation of epithelial cells during NPWT has been found to decrease (19). Cytokeratin (CK) is a member of intermediate filaments found in the intra-cytoplasmic cytoskeleton of epithelial tissue. It is well known that CK plays a critical role in cell structure integrity, and the expression of $\mathrm{CK}$ is remarkably tissue- and differentiation-specific. Each type of epithelium has its specific pattern of CK expression. CK10 is a specific keratinization marker; CK14 is a marker of basal cells layer; CK19 is a biochemical marker of skin stem cells in vivo (24). Our data indicated that NP treatment caused more keratinized epithelium, but less basal cell layer and skin stem cells. Epithelial cells play a critical role in wound healing, and their migration across the wound tissue to form a keratinized epithelium between the wound and the environment is one of the most important processes in wound healing. This may lead to the efficient healing effect of NPWT. However, our data demonstrated that the infiltration of MSCs was decreased in the NP group, which may indicate some disadvantages of NPWT. Firstly, the major mechanisms of the effects of MSCs on the wound repair process are thought to be structural repair via cellular differentiation, immune modulation and the secretion of cytokines, which may promote angiogenesis and the recruitment of fibroblasts and other cells (25). The low level of MSC infiltration in wound tissue will lead to chronic wounds and will delay the repair process. In addition, another consideration in the repair of wounds is the formation of scars, caused by deposition of excess ECM by fibroblasts in the wound bed. The cytokines and growth factors secreted by MSCs have been proved to reduce scar formation at the site of injury (26). The low level of MSCs infiltration in wound tissue will lead to an increased scar formation. Accordingly, although NPWT in promoting wound healing has been largely accepted by clinicians, the number of high-level clinical studies demonstrating its effectiveness is still limited (27). Thus, our data may indicate some disadvantages of NPWT that may need to be improved.

Collagen deposition was also influenced by NP and PP treatment. The significantly increased levels of collagen I in the NP group indicated that NP promoted the maturation of wounds on day 14. In addition, the significant increase in MMP-13 levels and the decrease in TIMP-1/2 levels on days 3 or 7 in the NP vs. the PP group may be helpful for completing the matrix remodeling in the early stages of healing, which may promote the wound healing effect.

In conclusion, our study demonstrates that NP is more effective than PP for wound healing by promoting the inflammation during the early stages of healing, increasing proliferation in the wound tissue, increasing the number of endothelial cells, epithelial cells and fibroblasts, and strengthening the remodeling process and matrix maturation.

\section{Acknowledgements}

This study was supported by the Key Projects of the Fund of Science and Technology Department of Hunan Province (2014SK2018) and the Scientific Research Fund of the Health Department of Hunan Province (132013-026).

\section{References}

1. Watterson KR, Lanning DA, Diegelmann RF and Spiegel S: Regulation of fibroblast functions by lysophospholipid mediators: Potential roles in wound healing. Wound Repair Regen 15: 607-616, 2007.

2. Ko SH, Nauta A, Wong V, Glotzbach J, Gurtner GC and Longaker MT: The role of stem cells in cutaneous wound healing: What do we really know? Plast Reconstr Surg 127 (Suppl 1): 10S-20S, 2011

3. Evans ND, Oreffo RO, Healy E, Thurner PJ and Man YH: Epithelial mechanobiology, skin wound healing, and the stem cell niche. J Mech Behav Biomed Mater 28: 397-409, 2013.

4. Maxson S, Lopez EA, Yoo D, Danilkovitch-Miagkova A and Leroux MA: Concise review: Role of mesenchymal stem cells in wound repair. Stem Cells Transl Med 1: 142-149, 2012.

5. Darby IA and Hewitson TD: Fibroblast differentiation in wound healing and fibrosis. Int Rev Cytol 257: 143-179, 2007. 
6. Nuschke A: Activity of mesenchymal stem cells in therapies for chronic skin wound healing. Organogenesis 10: 29-37, 2014.

7. Shi J, Xi W, Yi C, Wang Z, Guo S and Han Y: Vacuum sealing drainage promotes experimental pig explosive abdomen wound healing. Xi Bao Yu Fen Zi Mian Yi Xue Za Zhi 30: 312-315, 2014 (In Chinese).

8. Argenta LC, Morykwas MJ, Marks MW, DeFranzo AJ Molnar JA and David LR: Vacuum-assisted closure: State of clinic art. Plast Reconstr Surg 117 (Suppl): 127S-142S, 2006.

9. Banwell $\mathrm{P}$ and Téot L: Topical negative pressure (TNP): The evolution of a novel wound therapy. J Tissue Viability 16: 16-24 2006.

10. Morykwas MJ, Simpson J, Punger K, Argenta A, Kremers L and Argenta J: Vacuum-assisted closure: State of basic research and physiologic foundation. Plast Reconstr Surg 117 (Suppl): 121S-126S, 2006.

11. Banwell PE: Topical negative pressure therapy in wound care. $\mathrm{J}$ Wound Care 8: 79-84, 1999.

12. Fonder MA, Lazarus GS, Cowan DA, Aronson-Cook B Kohli AR and Mamelak AJ: Treating the chronic wound: A practical approach to the care of nonhealing wounds and wound care dressings. J Am Acad Dermatol 58: 185-206, 2008.

13. Hunter JE, Teot L, Horch R and Banwell PE: Evidence-based medicine: Vacuum-assisted closure in wound care management. Int Wound J 4: 256-269, 2007.

14. Chen SZ, Li J, Li XY and Xu LS: Effects of vacuum-assisted closure on wound microcirculation: An experimental study. Asian J Surg 28: 211-217, 2005.

15. Evans D and Land L: Topical negative pressure for treating chronic wounds: A systematic review. Br J Plast Surg 54: $238-242,2001$

16. Kairinos N, Solomons M and Hudson DA: The paradox of negative pressure wound therapy - in vitro studies. J Plast Reconstr Aesthet Surg 63: 174-179, 2010.

17. Muller MJ, Hollyoak MA, Moaveni Z, Brown TL, Herndon DN and Heggers JP: Retardation of wound healing by silver sulfadiazine is reversed by Aloe vera and nystatin. Burns 29: 834-836, 2003.
18. Greenhalgh DG: The role of apoptosis in wound healing. Int J Biochem Cell Biol 30: 1019-1030, 1998

19. Nuutila K, Siltanen A, Peura M, Harjula A, Nieminen T, Vuola J, Kankuri E and Aarnio P: Gene expression profiling of negative-pressure-treated skin graft donor site wounds. Burns 39: 687-693, 2013

20. Baldwin C, Potter M, Clayton E, Irvine L and Dye J: Topical negative pressure stimulates endothelial migration and proliferation: A suggested mechanism for improved integration of Integra. Ann Plast Surg 62: 92-96, 2009.

21. Potter MJ, Banwell P, Baldwin C, Clayton E, Irvine L, Linge C, Grobbelaar AO, Sanders R and Dye JF: In vitro optimisation of topical negative pressure regimens for angiogenesis into synthetic dermal replacements. Burns 34: 164-174, 2008

22. McNulty AK, Schmidt M, Feeley T and Kieswetter K: Effects of negative pressure wound therapy on fibroblast viability, chemotactic signaling, and proliferation in a provisional wound (fibrin) matrix. Wound Repair Regen 15: 838-846, 2007.

23. Lu F, Ogawa R, Nguyen DT, Chen B, Guo D, Helm DL, Zhan Q, Murphy GF and Orgill DP: Microdeformation of three-dimensional cultured fibroblasts induces gene expression and morphological changes. Ann Plast Surg 66: 296-300, 2011.

24. Michel M, Török N, Godbout MJ, Lussier M, Gaudreau P, Royal A and Germain L: Keratin 19 as a biochemical marker of skin stem cells in vivo and in vitro: Keratin 19 expressing cells are differentially localized in function of anatomic sites, and their number varies with donor age and culture stage. J Cell Sci 109: 1017-1028, 1996.

25. Balaji S, Keswani SG and Crombleholme TM: The role of mesenchymal stem cells in the regenerative wound healing phenotype. Adv Wound Care (New Rochelle) 1: 159-165, 2012.

26. Ankrum J and Karp JM: Mesenchymal stem cell therapy: Two steps forward, one step back. Trends Mol Med 16: 203-209, 2010.

27. Huang C, Leavitt T, Bayer LR and Orgill DP: Effect of negative pressure wound therapy on wound healing. Curr Probl Surg 51: 301-331, 2014. 